\title{
The ATLAS Tile Calorimeter Performance and its upgrade toward High-Luminosity LHC
}

\author{
Louis Vaslin ${ }^{a, *}$ on behalf of the ATLAS Tile Calorimeter Group \\ Laboratoire de Physique de Clermont, \\ 4 Avenue Blaise Pascal, 63178 Aubière Cedex, France \\ E-mail: louis.vaslin@clermont.in2p3.fr
}

The Tile Calorimeter is a hadronic calorimeter covering the central region of the ATLAS experiment. The signals are read-out by wavelength shifting fibres coupled to the photomultiplier tubes. The front-end electronics process those signals from which the energies are inferred. Each stage of the inference is calibrated using the calibration systems of the Tile Calorimeter. The performance of the calorimeter has been measured and monitored using calibration data, cosmic ray muons and the collision data acquired during LHC Run 1 and Run 2. The High-Luminosity phase of LHC (HL-LHC), is expected to begin in 2028. The electronics of the calorimeter will be replaced to cope with the higher trigger rate, radiation level and pile-up conditions. Prototypes of this new electronics were tested within a demonstrator module both in test beam and calibration runs. The performance and calibration of the Tile Calorimeter are summarized, as well as the results obtained from the test of the new electronics prototypes.

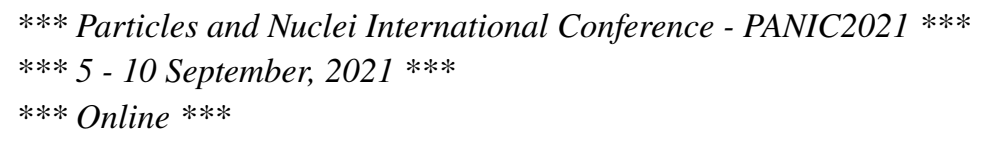

${ }^{*}$ Speaker 


\section{Introduction}

The ATLAS experiment [1] is one of the four main experiments located around the Large Hadron Collider [2] (LHC) at CERN. It is composed of several sub-detectors, including trackers, calorimeters and muon chambers. Within a few years, the LHC will enter its High-Luminosity phase. With this accelerator upgrade will also come several upgrades on the detectors side.

This paper will focus on the calibration and performance of the Tile calorimeter [3] (TileCal) of the ATLAS detector, as well as on the upcoming upgrade for HL-LHC [4]. In particular, the architecture and performance of the current system will be described as well as the planned upgraded system. Then, using the Tile demonstrator as a showcase, the performance of the early versions of the new components will be assessed.

\section{The ATLAS Tile Calorimeter}

The Tile Calorimeter is the central hadronic calorimeter of the ATLAS experiment. It is composed of 4 readout partitions of 64 modules each. In total, there are about 10000 channels to measure energy deposits up to $2 \mathrm{TeV}$. The partitions of the long barrel cover a pseudo-rapidity range $0<|\eta|<1.0$ and the end-cap barrel partitions cover a pseudo-rapidity range $0.8<|\eta|<1.7$. The calorimeter uses scintillating tile as active material and steel as absorber. The light produced in the scintillating tiles is collected through wavelength shifting optical fibers and guided toward PMTs.

\subsection{Structure and electronics of the Tile Calorimeter}

Each module is split into several cells, each cell being connected to two PMTs that are placed inside the Super Drawers (SDs) that contain the front-end electronics. The SDs are split into two interconnected drawers. The front-end electronics that can be found inside the SDs is composed of several components.

The 3-in-1 cards collect and amplify and shape the signal received from the PMTs. There are two amplification gains with a ratio of 1/64. The cards also have an integrator channel used for calibration with ${ }^{137} \mathrm{Cs}$ radioactive source and luminosity measurement. The digitizer cards digitize the shaped pulse in 7 samples taken every 25 ns using 10-bits ADCs. The digitized pulses are then stored in memory pipelines until the reception of a trigger signal. The interface cards handle the formatting of the data and send them to the back-end electronics with a maximum rate of $100 \mathrm{kHz}$. They also distribute the LHC clock to the modules as well as the configuration instructions. The adder cards are directly connected to the 3 -in-1. They compute an analogical sum of the signal to be used for the trigger. It is configured to identify energy deposits corresponding to jets and muons.

The data sent by the detector is then processed to retrieve the samples' signal pulse characteristics. From these characteristics, the corresponding energy deposit can be assessed using the calibration.

\subsection{Calibration}

The calibration system of TileCal is composed of three main components. The Cesium system uses a ${ }^{137} \mathrm{Cs}$ radioactive source that travels inside the modules of the calorimeter. The energy deposited by the source allows to monitor and calibrate the optical parts as well as the gain of the 
PMTs with a precision of $0.3 \%$. The laser system sends laser pulses directly to the PMTs. This system allows monitoring the variations from the PMTs and electronics with a precision of $0.5 \%$. The Charge Injection System (CIS) generate a known charge inside the 3-in-1 card. The electronics then process the resulting current to calibrate the ADC response with a precision of $0.7 \%$.

There is also a Minimum Bias system (MB) that uses the integrator readout to integrate over hundreds thousand events. The obtained results are then proportional to the luminosity of the LHC [5] and can be used to monitor the full optical chain (scintillators, optical fibers and PMTs). The electromagnetic energy scale to convert the ADC signals into energy is determined using electron beams of well-known energies produced at CERN test beam facility. The final calibration is then given by the Eq. (1) below.

$$
E[G e V]=A[A D C] \times f_{\mathrm{ADC} \rightarrow \mathrm{pC}} \times f_{\mathrm{pC} \rightarrow \mathrm{GeV}} \times f_{\mathrm{Cs}} \times f_{\mathrm{las}}
$$

In this equation, we can identify the energy scale constant $f_{\mathrm{ADC} \rightarrow \mathrm{pC}}$, the charge injection constant $f_{\mathrm{pC} \rightarrow \mathrm{GeV}}$, the Cesium constant $f_{\mathrm{Cs}}$ and the laser constant $f_{\text {las }}$.

\subsection{Performance during Run 2}

The defective channels that can impact the physics results are masked. The evolution of the portion of masked channels and cells can be monitored as shown on Figure 1. Since each cell is connected to two PMTs, the effective number of masked cells is smaller than the number of masked cells. The results show that the number of masked cells was kept under $1 \%$ during Run 2 . The short breaks during this period correspond to maintenance campaigns during which the most problematic channels were fixed. At the end of Run 2, only $0.48 \%$ of the cells were masked.

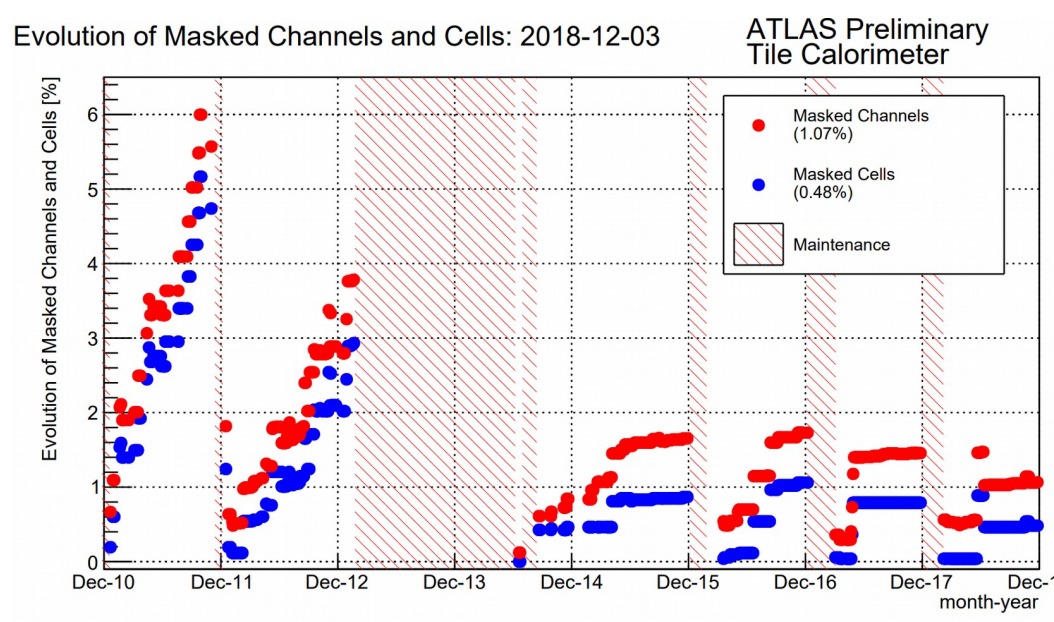

Figure 1: Evolution of the portion of masked channels and cells during Run 1 and Run 2 [4].

The response to cosmic muons can also be used to monitor the electromagnetic scale and cell intercalibration. The uniformity of the detector response across the $\eta$ range is better than 5\% and the uniformity across the azimuth angle $\phi$ better than $1 \%$. For the response to single hadrons the obtained energy-momentum ratios are in agreement with Monte Carlo simulations within 5\%. 


\section{Upgrade toward High Luminosity LHC}

In the future, the LHC will be upgraded toward its High-Luminosity phase (HL-LHC) during Long Shutdown 3 (2025-2027). It will lead to an increase of peak luminosity by a factor 7.5 compare to LHC nominal value, with up to $4000 \mathrm{fb}^{-1}$ integrated luminosity at the end of operations. As consequence of the higher luminosity an augmentation of the number of collisions per bunch crossing, also called pile-up, is also expected. In order to face these new challenges, the TileCal system will be upgraded with a new mechanical structure and electronics. It will also feature a new digital trigger, with a communication rate of up to $40 \mathrm{MHz}$.

\subsection{New structure and electronics}

The new SDs will be split into four fully independent mini drawers that contain up to 12 PMTs each. Since all mini drawers are independent, there will be a better segmentation of the detector for improved reliability and redundancy. The new mechanical architecture has been fully validated and production has already started. The electronics will also be completely replaced both on and off detector.

The front-end boards (FENICS) will replace the current 3-in-1 cards. They will have two gains with a ratio of $1 / 40$ for the signal channels as well as an integrator channel for luminosity measurement and Cesium calibration. The mainboard will handle the digitization with 12-bits ADCs for the signal and 16-bits ADC for the integrator channel. The daughterboard will handle the formatting of the data and send them to the off-detector electronics at a rate of $40 \mathrm{MHz}$. It will also manage the communication of the clock and of the instructions.

The data sent by the detector will be received by the back-end electronics called Tile PreProcessor. This data will undergo an online reconstruction and produce trigger object that will be used for the new digital trigger system. The new trigger will allow more advanced algorithms for improved efficiency at a higher rate. This is one of the most important features of the Tile Calorimeter upgrade in terms of physic results improvement. The Tile PreProcessor will also serve as an interface for the monitoring and remote control of the detector.

\subsection{Tile demonstrator}

The Tile demonstrator project was initiated in 2015 with the objective to operate early versions of the upgraded electronics using the new mechanical structure. It is a hybrid module that serves as a test case for the new components while ensuring compatibility with the current system. The demonstrator module is equipped with an modified version of the current 3 -in- 1 card with a gain ratio $1 / 32$ instead of 1/64 and uses a mainboard and daughterboard. In order to ensure the compatibility with the current trigger system, adder cards were also added to provide the analogical trigger signal. The demonstrator back-end electronics was also designed, so the module is fully compatible with the current control, monitoring and data acquisition systems. This module was inserted into the detector in 2019 with the objective to keep it inside the detector during Run 3. The study of the performances of the Tile demonstrator can give us some insight of what can be expected for HL-LHC. 


\subsubsection{Calibration run results}

Since the demonstrator module was inserted in the detector, several calibration runs have been taken during Long Shutdown 2 (LS2). We can use these data to see and compare the performances of the new module with the legacy ones.

The study of the data from laser calibration runs gives valuable information on the stability of the pulse characteristics. So we can study the variation of the mean pedestal for each channel for both the demonstrator and a legacy module, as well as the associated noise. It appears that the pedestal value obtained for the demonstrator is very stable, with a noise level lower than for legacy modules. These kinds of results demonstrate the performance of the new electronics with respect to the current system.

\section{Conclusion}

The Tile calorimeter of the ATLAS detector can produce valuable results. With the help of the good monitoring provided by the calibration systems and of the regular maintenance campaigns during Run 2, the number of masked cells was kept below $0.48 \%$ and the cell energy scale was maintained within $1 \%$. Ones should also note the extensive maintenance campaign carried during the duration of LS2 to ensure that the detector will be in the best condition at the beginning of Run 3 next year.

Nevertheless, with the upcoming High-Luminosity upgrade of the LHC, many new challenges will arise, among which an increased pile-up contribution and increased radiation level. In order to face these challenging conditions, the Tile calorimeter will also undergo a major upgrade. This upgrade will bring a new mechanical structure for the Super Drawers, a new on and off detector electronics, and most importantly a new digital trigger system that allows the use of more advanced trigger algorithms at a much higher rate (up to $40 \mathrm{MHz}$ ).

In order to test the future element of the Tile upgrade, a demonstrator module has been built and inserted in the detector. This hybrid module allows us to test early versions of the new electronics, while ensuring compatibility with the current trigger, monitoring and data acquisition systems. The results obtained with the demonstrator module show good performances both for test beams and calibration runs taken after insertion in the detector.

\section{References}

[1] ATLAS collaboration, The ATLAS Experiment at the CERN Large Hadron Collider, JINST 3 (2008) S08003.

[2] L.R. Evans and P. Bryant, LHC Machine, JINST 3 (2008) S08001.

[3] ATLAS collaboration, ATLAS tile calorimeter: Technical Design Report, Tech. Rep. CERN-LHCC-96-042, ATLAS-TDR-3, CERN, Geneva (1996).

[4] ATLAS collaboration, Technical Design Report for the Phase-II Upgrade of the ATLAS Tile Calorimeter, Tech. Rep. CERN-LHCC-2017-019, ATLAS-TDR-028, CERN, Geneva (Sep, 2017). 
[5] G. Aad, T. Abajyan, B. Abbott, J. Abdallah, S. Abdel Khalek, A.A. Abdelalim et al., Improved luminosity determination in pp collisions at $\sqrt{s}=7 \mathrm{TeV}$ using the atlas detector at the lhc, Eur. Phys. J. C 73 (2013) 2518. 